\title{
Apego, Conflito e Auto-Estima em Adolescentes de Famílias Intactas e Divorciadas
}

\author{
Attachment, Conflict and Self-Esteem in Adolescents from Intact \\ and Divorced Families
}

\author{
Catarina Pinheiro Mota* \& Paula Mena Matos* \\ Universidade do Porto
}

\begin{abstract}
Resumo
Este estudo teve como objectivo analisar a contribuição da estrutura familiar (intacta e divorciada), do conflito interparental, do apego aos pais e aos pares para a auto-estima do adolescente. A amostra foi constituída por 403 adolescentes, entre os 14 e os 19 anos de idade. A análise univariada da variância mostrou que a estrutura familiar não prediz a auto-estima. Contudo, os estilos de apego aos pais derivados das análises de clusters e baseados no modelo bidimensional de Bartholomew foram associados à autoestima, com níveis mais elevados para adolescentes de estilo seguro. A variância multivariada com o apego aos pais e aos pares como fatores independentes mostraram que o apego aos pares não desempenha um papel moderador na predição da auto-estima nos adolescentes. A regressão múltipla hierárquica indicou que uma elevada qualidade dos apegos aos pais e pares se mostram relevantes na predição da auto-estima.
\end{abstract}

Palavras-chave: Apego; Conflito interparental; Auto-estima; Adolescência; Configuração familiar.

\begin{abstract}
This study aimed to analyze the differential contribution to adolescent's self-esteem concerning family structure (intact and divorced families), interparental conflict, and attachment to parents and peers. The sample consisted of 403 adolescents, aged from 14 to 19. Univariate ANOVA showed that family structure does not predict self-esteem, but adolescents from intact families with higher levels of interparental conflict presented lower self-esteem. Parental attachment patterns derived from cluster analysis and based on Bartholomew bi-dimensional model were associated with self-esteem, namely, with higher levels for adolescents with a secure style. An ANOVA showed no moderation role in predicting adolescents' self-esteem, when attachment to parents and peers were considered as independent factors. On the other hand, multiple regression results indicated that high quality bonds to parents and peers are predictors of self-esteem.

Keywords: Attachment; Interparental conflict; Self-esteem; Adolescence; Family configuration.
\end{abstract}

No curso da sua evolução, a teoria do apego (Bowlby, 1980) tem vindo a aumentar o ímpeto das investigações sobre as relações primárias com figuras significativas e a exploração dos determinantes na qualidade dos relacionamentos. Mais recentemente a investigação tem prestado atenção ao impacto dessas relações na adolescência e fase jovem adulta com todas as vicissitudes inerentes ao ciclo vital. O processo vinculativo realça a importância dos laços afetivos precoces que se encarregam de criar uma base segura no ser humano. A capacidade para a formação de laços emocionais constitui um elemento de extrema relevância para o desenvolvimento e funcionamento psicológico, procurando contribuir para a com-

\footnotetext{
${ }^{*}$ Endereço para correspondência: Universidade do Porto, Faculdade de Psicologia e de Ciências da Educação, Rua Dr. Manuel Pereira da Silva, 4200-392 Porto, Portugal. Tel.: +351 22607 9700; Fax. +351 226079 725. E-mail: catarinap.mota@gmail.com; pmmatos@fpce.up.pt Trabalho realizado no âmbito do projecto da Fundação para a Ciência e Tecnologia- PTDC/PSI/65416/2006.
}

preensão das perturbações psicológicas que têm por base a dissolução ou ruptura indesejada dos laços afetivos (Matos, 2002). Desta forma, a disponibilidade e sensibilidade pessoal dos pais ou figuras cuidadoras constitui um preditor para o desenvolvimento futuro de laços afetivos, constatando-se uma associação entre a segurança emocional e as relações com o grupo de pares e par amoroso (e.g. Allen et al., 2003). Na perspectiva do ciclo vital, o apego surge como um processo contínuo que não termina na infância. Esta fase percebe-se como difícil para o adolescente que, por um lado, busca a sua independência psicológica e a sua autonomia pelo afastamento, mas por outro lado, não sente suficiente segurança para se separar dos pais, recorrendo a eles como fonte de apoio (Matos \& Costa, 1996). Nesta medida, torna-se de extrema importância para o jovem viver num clima de segurança e afeto. Actualmente os jovens atravessam transições familiares tais como o divórcio dos pais, contexto no qual, por vezes, suscitam dúvidas acerca da manutenção destes laços afetivos; criando-se a ideia 
aparente de que a separação parental promove frequentemente uma ineficácia no desenvolvimento do processo adaptativo e resiliente dos jovens.

Em Portugal, o surto de divórcios teve o seu início no final dos anos 60, crescendo progressivamente pelos anos 80 , atingindo um pico bastante elevado, embora mais estável, nos anos 90 (Torres, 1996). Nos últimos anos projeta-se que aproximadamente metade das crianças que nascem no seio de pais casados irão experimentar o divórcio dos pais antes de atingir a idade de 18 anos (Goodman \& Emery, 1998). O divórcio implica separação e perda, especialmente no que se refere a um self relacional. Esta transição origina inevitavelmente um impacto a nível de toda a família, incluindo os filhos que tendem a partilhar as emoções intensas e entram igualmente num processo de luto e reorganização do equilíbrio psicológico (Matos \& Costa, 2004). Assim, os efeitos do divórcio dependem da forma como o casal gere a separação, pelo que a presença ou ausência de conflitos interparentais influencia a adaptatividade do jovem face à situação. Quando a separação implica um movimento para uma maior harmonia e menor constrangimento familiar, os resultados podem inclusive ser mais satisfatórios para os jovens sob o ponto de vista do seu bem-estar, quando comparados com famílias não divorciadas com um elevado conflito (Amato \& Sobolewski, 2001). Assim sendo, merece a nossa atenção a análise dos fatores comuns que podem dificultar o ajustamento dos jovens, como sejam a relação familiar, a comunicação e o conflito parental, nos períodos pré e pós-divórcio. É sabido que muitos dos problemas exibidos pelos jovens resultam da existência de conflitos familiares, mesmo antes de haver qualquer tipo de re-estruturação familiar (Emery \& Forehand, 1996). Jovens expostos a elevados níveis de conflitos interparentais apresentam dificuldades no processo de ajustamento que podem passar por internalização ou externalizaçao de problemas. Assim, um fator contextual que afeta a apreciação do jovem face aos conflitos interparentais é a qualidade afetiva do relacionamento que estabelece com cada um dos pais ou figuras cuidadoras (Cox, Paley, \& Hater, 2001). Desta forma, o predomínio de afeto negativo dos pais (como estarem zangados, exaustos e desmoralizados), em resultado do conflito conjugal, acaba por alterar as interacções entre os pais e os jovens. A indisponibilidade sob o ponto de vista emocional poderá levar a que as necessidades emocionais dos jovens não sejam percebidas ou detectadas (Cox et al., 2001). Neste sentido, Noller (1994) analisa as relações entre o funcionamento familiar e diferentes dimensões do auto-conceito (onde se inclui a auto-estima), constatando a existência de uma relação positiva entre os níveis de afeto e qualidade no relacionamento familiar e a auto-estima do jovem. Ainda segundo o mesmo autor, a questão reside entre as relações com os pares e a importância das relações familiares no período de desenvolvimento da adolescência. A qualidade do funcionamento familiar torna possível a construção de sentimentos de competência e valor, contribuindo para o de- senvolvimento de uma auto-estima positiva (Peixoto, 2004). O presente estudo procura focalizar a importância das dimensões relacionais estabelecidas com as figuras significativas, tendo em conta, por um lado, a qualidade das relações com os pais e o nível de conflito interparental, e por outro lado, a relação com o grupo de pares, tentando perceber a sua influência na auto-estima dos adolescentes.

\section{Objetivos}

Este estudo pretende analisar a contribuição da configuração familiar (intacta e divorciada), do conflito interparental e da vinculação aos pais e aos pares, para a predição da auto-estima do adolescente. Também se objetiva examinar o papel moderador da vinculação aos pares na associação entre a vinculação aos pais e a auto-estima.

\section{Hipóteses}

Frente aos objetivos propostos, espera-se que, independentemente da configuração familiar a qualidade nos laços vinculativos aos pais e aos pares esteja significativamente associada à auto-estima. Ao mesmo tempo, espera-se que o conflito interparental seja melhor preditor da vinculação aos pais e da auto-estima do que a estrutura familiar. Também espera-se que a vinculação aos pais se associe à auto-estima sem que exista um efeito moderador da vinculação aos pares.

\section{Método}

\section{Participantes}

O estudo foi composto por 403 adolescentes, entre os 14 e os 19 anos de idade $(M=16,81 ; D P=1,10), 234$ do gênero feminino e 169 do gênero masculino. A maioria $(n=350)$ provinha de famílias intactas e 53 de famílias divorciadas.

\section{Instrumentos}

Dados demográficos. Foi usada uma ficha demográfica, onde se incluíram informações referentes ao gênero, idade, identificação familiar (idade, profissão, escolaridade e estado civil das figuras parentais), identificação escolar e projectos futuros, bem como informação relativa a questões do divórcio.

Auto-Estima. Foi usada a Rosenberg's Self-Esteem Scale (Rosenberg, 1979; Adaptação de Rocha \& Matos, 2003) para medir a auto-estima dos adolescentes participantes. É uma escala de 10 itens do tipo Likert de 6 pontos de avaliação qualitativa. $\mathrm{O}$ alfa de Cronbach para a presente amostra foi .86. A análise fatorial confirmatória apresentou índices de ajustamento adequados $(\mathrm{RMR}=.029$; $\mathrm{GFI}=.952 ; \mathrm{AGFI}=.914 ; \mathrm{CFI}=.963 ; \mathrm{RMSEA}=.072$ ).

Apego. Foi usado o Questionário de Vinculação ao Pai e à Mãe - QVPM (Matos \& Costa, 2001) para se medir o apego dos adolescentes e jovens adultos aos seus pais, o qual possui 30 itens que traduzem afirmações sobre as relações familiares. Focaliza três dimensões: a Qualidade do laço emocional (QLE), a Ansiedade de separação 
(AS) e, por último, a Inibição da exploração e individualidade (IEI). A resposta é tipo Likert de seis pontos, realizada para o Pai e Mãe, respectivamente. Os alfas de Cronbach para a presente amostra foram: IEI pai $=.81$; IEI mãe=.82; QLE pai $=.94$; QLE mãe=.92; AS pai $=.85$; AS mãe=.83. As análises fatoriais confirmatórias apresentam índices de ajustamento adequados para os modelos $($ RMR pai $/$ mãe $=.068 / .047 ;$ GFI pai $/$ mãe $=.964 / .967$; AGFI pai $/ \mathrm{mãe}=.922 / .927$; CFI pai $/$ mãe $=.979 / .975$; RMSEA pai $/$ mãe $=.072 / .074$ ).

Para medir o apego dos participantes aos pares, foi usada a versão Pares do Inventory of Peer and Parental Attachment - IPPA (Armsden \& Greenberg, 1987; Adaptação de Ferreira \& Costa, 1998). Os 25 itens enfocam 3 dimensões: Confiança; Comunicação; e Alienação. O formato de resposta é tipo Likert com 6 pontos. Os alfas de Cronbach para a presente amostra foram: Confiança= .86; Comunicação=.85 e Alienação= .82. A análise fatorial confirmatória apresenta índices de ajustamento adequados para o modelo $(\mathrm{RMR}=.029 ; \mathrm{GFI}=.967$; AGFI=.929; CFI $=.975 ;$ RMSEA $=.074)$.

Conflito parental. Os participantes relataram sua percepção do conflito parental usando o Childrens' Perception of Interparental Conflict Scale (Grych, Seid, \& Fincham, 1992; Adaptação de Andrade \& Matos, 2003). Embora o instrumento tenha originalmente 49 itens, com 9 dimensões, para o presente estudo, foi usada apenas uma parte da escala com vista a avaliar as propriedades do conflito interparental, pelo que só os itens referentes à frequência, intensidade e resolução constaram na escala, ficando por isso reduzida a 19 itens. Cada item utiliza uma escala tipo Likert, com seis alternativas de resposta. Os alfas de Cronbach para a presente amostra foram: Frequência=.82; Resolução=.88 e Intensidade $=.86$. A análise fatorial confirmatória apresenta igualmente índices de ajustamento adequados para o modelo $(\mathrm{RMR}=.041 ; \mathrm{GFI}=.981 ; \mathrm{AGFI}=.922 ; \mathrm{CFI}=.986$; RMSEA=.089).

\section{Procedimento}

Foi obtido um Consentimento Informado dos participantes conforme requerido pelo Conselho Executivo de cada escola, que aprovou o caráter ético da pesquisa. Igualmente, foram assegurados a confidencialidade e o sigilo dos dados. Tratando-se de um estudo transversal, a coleta dos dados foi realizada num só momento em escolas secundárias da região norte e centro de Portugal. A aplicação teve lugar em tempo letivo, sendo apoiada pela equipa de investigação. Foram apresentados os objetivos gerais do estudo, pelo que, tratando-se de uma administração coletiva, foram dadas instruções padronizadas de esclarecimento no que diz respeito ao preenchimento dos questionários de auto-relato. A ordem dos questionários foi invertida aleatoriamente no intuito de evitar o enviesamento dos resultados derivados do factor cansaço e sequência dos instrumentos.

\section{Resultados}

Apesar do presente estudo conter dois objetivos principais, os resultados estão descritos em quatro fases, sendo que as três primeiras vão ao encontro do primeiro objetivo, e ainda parte da terceira e a quarta análise coadunam-se com o segundo objectivo.

\section{Análises Preliminares}

Diferenças entre os dois grupos (famílias intactas e divorciadas) foram testadas através de análises univariadas ANOVAS e Qui-quadrado, observando-se diferenças na escolaridade dos adolescentes que é ligeiramente menor em adolescentes provindos de famílias divorciadas $[F(1,395)=4,6 ; p=.033]$. Adolescentes de famílias divorciadas também foram reprovados mais vezes do que seus pares de famílias intactas $[F(1,389)=7,4 ; p=.007]$. A escolaridade das mães e dos pais dos adolescentes é significativamente superior no caso de famílias divorciadas $[F(1,395)=13,14 ; \mathrm{p}=.000]$ e $[F(1,373)=10,57$; $p=.001]$, respectivamente. Por último, existe ainda uma diferença significativa em relação à idade das mães, sendo menor nas mães de famílias divorciadas $[F(1,395)$ $=14,28 ; p=.000]$. Na amostra de adolescentes provindos de famílias divorciadas constata-se que os divórcios ocorrem desde o nascimento até aos 18 anos de idade do adolescente $(M=9,67 ; D P=5,4)$. Ainda face a adolescentes provenientes de famílias divorciadas, $72,3 \%$ vive com a mãe depois do divórcio, enquanto $8,5 \%$ vive em custódia conjunta, $10,6 \%$ só com o pai e $8,5 \%$ vive com outros (avós, tios, etc.), 22,4\% dos pais voltaram a casar, enquanto $11,8 \%$ das mães voltaram a casar. As variáveis que evidenciaram diferenças em função da pertença a famílias intactas ou divorciadas foram introduzidas subsequentemente nas análises como co-variantes.

\section{Efeito da Configuração Familiar e do Gênero na Auto-Estima}

A ANCOVA bifatorial realizada com a auto-estima em função da interação entre a configuração familiar e o gênero não foi significante. Isto significa que adolescentes de famílias intactas e divorciadas, independentemente do género, não diferem significativamente na auto-estima.

Efeito do Conflito Interparental e da Configuração Familiar na Auto-Estima. Foram então realizadas análises de covariância multivariada, mediante a análise diferencial em função do conflito interparental e da configuração familiar. Primeiramente efetuou-se a categorização do conflito interparental em dois níveis (alto e baixo) onde resultaram quatro grupos: Baixo conflito interparental em famílias intactas $(n=187)$; Alto conflito interparental em famílias intactas ( $n=166)$; Baixo conflito interparental em famílias divorciadas $(n=16)$ e Alto conflito interparental em famílias divorciadas $(n=33)$. A categorização realizada face aos dois níveis de conflito provém de um 
Mota, C. P. \& Matos, P. M. (2009). Apego, Conflito e Auto-Estima em Adolescentes de Famílias Intactas e Divorciadas.

valor obtido pela mediana do conflito interparental total, sendo baixo conflito se Mediana $\leq 2,86$ e alto conflito se Mediana $>2,86$. A ANCOVA bifactorial realizada permitiu constatar um efeito significativo do conflito $\mathrm{x}$ configuração familiar na auto-estima $[F(3,365)=8,34$; $p=.000]$. Tendo-se procedido ao teste post hoc de Sheffé para determinar a direção das diferenças, constata-se uma diferença significativa na auto-estima dos jovens entre o alto e baixo conflito em famílias intactas (ver
Tabela 1). Posteriormente foi medido o efeito do conflito interparental nas diferentes estruturas familiares sobre o apego aos pares. A MANCOVA revela que o conflito interparental x configuração familiar afecta significativamente o apego aos pares $[F(9,1164)=4,252$; $p=.000]$, nas dimensões de alienação $[F(3,388)=5,125$; $p=.002]$ e confiança $[F(3,388)=6,693 ; p=.000]$. O teste de Sheffé aponta para uma diferença significativa na alienação e confiança entre o alto e baixo conflito em famílias intactas (ver Tabela 1).

Tabela 1

Médias e Desvios Padrão para a Auto-estima, os Pares e os Pais em Função do Conflito Interparental e da Estrutura Familiar

\begin{tabular}{|c|c|c|c|c|c|c|c|c|c|}
\hline & \multicolumn{2}{|c|}{$\begin{array}{c}\text { Famílias intactas } \\
\text { com baixo conflito } \\
\text { interparental } \\
n=187\end{array}$} & \multicolumn{2}{|c|}{$\begin{array}{l}\text { Famílias intactas } \\
\text { com alto conflito } \\
\text { interparental } \\
n=166\end{array}$} & \multicolumn{2}{|c|}{$\begin{array}{c}\text { Famílias divorciadas } \\
\text { com baixo conflito } \\
\text { interparental } \\
n=16\end{array}$} & \multicolumn{2}{|c|}{$\begin{array}{c}\text { Famílias } \\
\text { divorciadas } \\
\text { com alto conflito } \\
\text { interparental } \\
n=33\end{array}$} & \multirow[t]{2}{*}{$\begin{array}{l}\text { Diferenças } \\
\text { Significativas } \\
\quad * * *\end{array}$} \\
\hline & $M$ & $D P$ & $M$ & $D P$ & $M$ & $D P$ & $M$ & $D P$ & \\
\hline Auto-Estima & 4.72 & .76 & 4.34 & .81 & 4.63 & 1.1 & 4.31 & .95 & $1>2$ \\
\hline IPPAComunicação & 4.85 & .69 & 4.65 & .87 & 4.72 & .63 & 4.88 & .63 & \\
\hline Alienação & 2.51 & .94 & 2.93 & 1.11 & 2.76 & .88 & 2.73 & .97 & $2>1$ \\
\hline Confiança & 5.03 & .67 & 4.68 & .84 & 5.13 & .58 & 4.87 & .62 & $1>2$ \\
\hline QVPM PAIIEI & 2.78 & .83 & 3.34 & .931 & 2.77 & .58 & 3.26 & .96 & $1<4 ; 1<2$ \\
\hline$Q L E$ & 5.31 & .59 & 4.58 & 1.00 & 4.68 & .84 & 3.81 & 1.50 & $1>2 ; 1>4 ; 2>4$ \\
\hline$A S$ & 4.02 & .86 & 3.75 & .95 & 3.41 & 1.1 & 3.22 & 1.12 & $1>4 ; 2>4$ \\
\hline QVPM MÃE IEI & 2.92 & .88 & 3.46 & .99 & 2.80 & .70 & 3.44 & .86 & $2>1$ \\
\hline$Q L E$ & 5.40 & .53 & 4.74 & 1.02 & 5.02 & .79 & 4.63 & 1.22 & $1>2 ; 1>4$ \\
\hline$A S$ & 4.06 & .85 & 3.84 & .96 & 3.71 & 1.33 & 3.89 & 1.02 & \\
\hline
\end{tabular}

Nota. IEI - Inibição da Exploração e Individualidade; QLE - Qualidade do Laço Emocional; AS- Ansiedade de Separação. $* * * p<.001$

Ainda nesta fase do estudo foi medido o efeito do conflito interparental nas diferentes configurações familiares sobre o apego aos pais. A MANCOVA permitiu constatar um efeito significativo do conflito $\mathrm{x}$ configuração familiar no apego aos pais $[F(18,1041)=6,86$; $p=.000]$, tanto a nível do pai como da mãe. Contudo, enquanto no pai existe uma significância em todas as dimensões, isto é, Inibição da Exploração e Individualidade $[F(3,350)=11,287 ; p=.000]$, Qualidade do Laço Emocional $[F(3,350)=32,859 ; p=.000]$ e Ansiedade de Separação $[F(3,350)=7,454 ; p=.000]$; no caso da mãe existe apenas para a Inibição da Exploração e Individualidade $[F(3,350)=10,122 ; p=.000]$ e para a Qualidade do Laço Emocional $[F(3,350)=18,748 ; p=.000]$. Mediante a análise com o teste post hoc (Sheffé), constatou-se uma diferença significativa em várias dimensões. Começamos por descrever a diferença encontrada na IEI pai e mãe face ao alto e baixo conflito nas famílias intactas, sendo que o IEI é maior nas famílias intactas de alto conflito $(M=3,34 ; D P=.931 ; M=3,46 ; D P=.988$, respectivamente para pai e mãe), comparativamente com as famílias intactas de baixo conflito $(M=2,78 ; D P=.825 ; M=2,92$; $D P=.884$, respectivamente para pai e mãe). Note-se que para o pai foi possível observar ainda uma diferença significativa entre famílias intactas e divorciadas, pelo que se constatou maior IEI em famílias divorciadas de elevado conflito interparental $(M=3,26 ; D P=.960)$, comparativamente a famílias intactas de baixo conflito interparental $(M=2,78 ; D P=.825)$ (ver Tabela 1). Quanto à QLE é significativamente maior quando existe um baixo conflito nas famílias intactas $(M=5,31 ; D P=.590$; $\mathrm{M}=5,40 ; D P=.525$, para pai e mãe), comparativamente com um alto conflito nas famílias intactas $(M=4,58$; $D P=1.004 ; M=4,74 ; D P=1.023$, para pai e mãe), assim como, comparativamente com o alto conflito em famílias divorciadas $(M=3,81 ; D P=1.503 ; M=4,63 ; D P=1.227$, respectivamente para pai e mãe). Ainda em relação à QLE observamos uma diferença significativa apenas constatada na relação com o pai, sendo que a QLE é maior em famílias intactas de alto conflito $(M=4,58 ; D P=1.004)$, comparativamente com famílias divorciadas com alto conflito $(M=3,81 ; D P=1.503)$ (ver Tabela 2). Por último 
existem diferenças significativas apenas para a relação com o pai na AS, encontrando-se valores superiores em famílias intactas de alto e baixo conflito, respectivamente $(M=3,75 ; D P=.950$ e $M=4,02 ; D P=.855)$ comparando com famílias divorciadas com elevado conflito $(M=3.22$; $D P=1.121)$ (ver Tabela 1).

Estilos de Apego às Figuras Parentais. Na terceira parte do presente estudo, foi realizada uma análise de clusters no intuito de verificar em que medida os padrões dos resultados que definem os diferentes clusters são consistentes com o modelo bi-dimensional de avaliação do apego de Bartholomew (Bartholomew \& Horowitz, 1991). No presente estudo foi usado um método combinatório; hierárquico e não-hierárquico ( $K$-means), no sentido de conseguir os benefícios de ambos os métodos (Hair, Anderson, Tatham \& Black, 1998). Através da técnica hierárquica foram especificados os centróides que, posteriormente, através do método não-hierárquico ( $K$ means), serviram de ponto de partida para a criação de clusters. De acordo com os valores obtidos nas dimen- sões, procedeu-se a uma leitura dos clusters de acordo com os quatro protótipos de apego propostos por Bartholomew (Bartholomew \& Horowitz, 1991), a saber, Seguro, Preocupado, Amedrontado e Desinvestido. Foram realizadas MANOVAS, que permitiram observar a variabilidade de cada uma das dimensões em função do cluster, confirmando a correspondência com o protótipo de apego. Na versão Pai constata-se assim um efeito significativo dos clusters $[F(9,1080)=93,106 ; p=.000]$. Todas as dimensões do QVPM Pai têm significância $(p=.000)$, exceto nas dimensões de Inibição da Exploração e Individualidade entre os clusters Desinvestido e Amedrontado e entre os clusters Seguro e Preocupado na dimensão de Ansiedade de Separação (ver Tabela 2). Na versão Mãe constatou-se igualmente um efeito significativo dos clusters $[F(9,1086)=92,450 ; p=.000]$. Todas as dimensões do QVPM Mãe têm significância $(p=.000)$, exceto nas dimensões de Inibição da Exploração e Individualidade entre os clusters Desinvestido e Preocupado e entre os clusters Seguro e Preocupado na dimensão de Qualidade do Laço Emocional (ver Tabela 2).

Tabela 2

Médias das Dimensões do QVPM, Auto-Estima e IPPA em Função dos Clusters para o Pai e a Mãe

\begin{tabular}{|c|c|c|c|c|c|}
\hline & $\begin{array}{l}\text { CLUSTER } 1 \\
\text { Desinvestido }\end{array}$ & $\begin{array}{l}\text { CLUSTER } 2 \\
\text { Seguro }\end{array}$ & $\begin{array}{l}\text { CLUSTER } 3 \\
\text { Amedrontado }\end{array}$ & $\begin{array}{l}\text { CLUSTER } 4 \\
\text { Preocupado }\end{array}$ & $\begin{array}{l}\text { Diferenças } \\
\text { Significativas }\end{array}$ \\
\hline PAI $n$ & 23 & 130 & 106 & 105 & \\
\hline$Q V P M \quad I E I$ & 3.31 & 2.21 & 3.27 & 3.79 & $1>2 ; 4>1 ; 4>3 ; 3>2$ \\
\hline$Q L E$ & 2.18 & 5.54 & 4.28 & 5.23 & $2>1 ; 2>3 ; 2>4 ; 4>3 ; 3>1 ; 4>1$ \\
\hline$A S$ & 1.95 & 4.31 & 3.16 & 4.31 & $2>1 ; 2>3 ; 3>1 ; 4>1 ; 4>3$ \\
\hline Auto-Estima & 4.01 & 4.79 & 4.36 & 4.42 & $2>1 ; 2>3 ; 2>4$ \\
\hline IPPA Comunicação & 4.73 & 4.93 & 4.54 & 4.84 & $2>4$ \\
\hline Alienação & 2.83 & 2.40 & 2.89 & 2.85 & $3>2 ; 4>2$ \\
\hline Confiança & 4.72 & 5.05 & 4.69 & 4.89 & $2>4$ \\
\hline MAE $n$ & 48 & 126 & 106 & 86 & \\
\hline QVPM IEI & 3.42 & 2.23 & 3.96 & 3.49 & $3>2 ; 3>1 ; 3>4 ; 4>2 ; 1>2$ \\
\hline$Q L E$ & 3.47 & 5.57 & 4.73 & 5.59 & $2>3 ; 2>1 ; 4>3 ; 4>1$ \\
\hline$A S$ & 2.51 & 4.07 & 3.74 & 4.80 & $4>2 ; 4>3 ; 4>1 ; 3>1 ; 2>3 ; 2>1$ \\
\hline Auto-Estima & 4.10 & 4.78 & 4.34 & 4.48 & $2>3 ; 2>1$ \\
\hline IPPA Comunicação & 4.40 & 4.82 & 4.66 & 5.00 & $4>3 ; 4>1$ \\
\hline Alienação & 2.64 & 2.39 & 3.00 & 2.73 & $2<3$ \\
\hline Confiança & 4.65 & 4.98 & 4.75 & 4.99 & \\
\hline
\end{tabular}

Nota. IEI - Inibição da Exploração e Individualidade; QLE - Qualidade do Laço Emocional; AS- Ansiedade de Separação. *** $p<.001$.

Desta forma, os resultados obtidos na análise anterior permitiram associar os clusters obtidos a cada protótipo de vinculação de acordo com a descrição realizada por Bartholomew (1990; Bartholomew \& Horowitz, 1991), tendo em conta as dimensões de vinculação aos pais de Matos e Costa (2001). Posto isto, o cluster 1 pareceu incluir o grupo de sujeitos Desinvestidos ao pai e à mãe, apresentando valores baixos de Qualidade do Laço
Emocional e de Ansiedade de Separação. Este grupo está descrito como tendo uma percepção positiva de si e negativa dos outros, sendo por isso independentes, evitando o relacionamento com os outros. Freqüentemente rejeitam o suporte dos pais, não evidenciando Ansiedade de Separação (Bartholomew \& Horowitz, 1991). No cluster 2 agruparam-se os sujeitos com protótipo de apego Seguro ao pai e à mãe, com valores reduzidos de 
Inibição da Exploração e Individualidade, valores elevados de Qualidade do Laço Emocional e médias de Ansiedade de Separação. Estes sujeitos têm uma representação positiva de si e dos outros, sentem-se confortáveis por depender dos outros, sem perder a autonomia aceitando o suporte dos pais (Bartholomew \& Horowitz, 1991). No cluster 3 agruparam-se os indivíduos com um padrão Amedrontado ao pai e à mãe, apresentando valores elevados de Inibição da Exploração e da Individualidade. A Qualidade do Laço Emocional, tal como a Ansiedade de Separação, apresentou valores abaixo dos indivíduos de padrão seguro, embora maior que os desinvestidos. Estes indivíduos detêm uma apresentação negativa de si e dos outros, evitam a proximidade e a intimidade com os outros, muito embora sejam bastante dependentes. Experienciam sentimentos de rejeição da parte dos pais, sendo extremamente críticos apesar de terem dificuldades na resolução da separação (Bartholomew \& Horowitz, 1991). Por último, o cluster 4 agrupou sujeitos com apego Preocupado ao pai e mãe, apresentando valores elevados de Qualidade de Laço Emocional, mais baixos que os indivíduos seguros (cluster 2). Estes indivíduos têm tendência à desvalorização de si próprios e têm uma percepção positiva dos outros; são dependentes, embora não sintam os outros tão próximos quanto gostariam. Caracterizam-se pela presença de um forte envolvimento emocional face aos pais, tendo elevada Ansiedade de Separação (Bartholomew \& Horowitz, 1991).

Diferenças no Apego aos pares e na Auto-Estima do Adolescente em Função da Distribuição dos Estilos de Apego ao Pai e à Mãe. Foram ainda realizadas MANOVAs com as dimensões do apego aos pares e a auto-estima em função da distribuição dos estilos de apego ao pai e à mãe. No que concerne ao apego aos pares, verificou-se um efeito principal dos clusters em todas as dimensões (comunicação, confiança e alienação), $[F(9$, $1053)=4,447 ; p=.000]$ e $[F(9,1059)=4,689 ; p=.000]$ respetivamente para o pai e a mãe. Para a versão Pai existe uma diferença significativa a nível das dimensões comunicação, confiança e alienação $[F(3,351)=6,042$; $p=.001],[F(3,351)=6,950 ; p=.000]$ e $[F(3,351)=4,956$; $p=.002]$, respetivamente. O cluster Seguro revela maiores níveis de Confiança e Comunicação comparativamente ao cluster Preocupado. Por outro lado, o cluster Preocupado e Amedrontado apresentam maiores níveis de Alienação comparativamente ao cluster Seguro. Para a versão Mãe existe uma diferença significativa a nível da Comunicação $[F(3,353)=6,352 ; p=.000]$, sendo maior no cluster Preocupado em comparação com o cluster Amedrontado e o cluster Desinvestido; a nível da Alienação existe uma diferença significativa $[F(3,353)=7,474$; $p=.000]$, sendo menor no cluster Seguro comparativamente com o Amedrontado (ver Tabela 2). Face à Autoestima, tanto para a versão Pai como para a Mãe constatou-se igualmente um efeito principal dos clusters $[F(3$,
$336)=10,131 ; p=.000]$ e $[F(3,339)=10,097 ; p=.000]$ respectivamente. Em relação à versão Pai verifica-se uma diferença significativa entre o cluster Seguro que apresenta valores mais elevados comparativamente com o cluster Desinvestido, Amedrontado e o Preocupado. Para a versão Mãe, existe uma diferença significativa entre o cluster Seguro que apresenta valores mais elevados comparativamente com o cluster Amedrontado e com o Desinvestido (ver Tabela 2).

Papel Moderador dos Pares no Apego aos Pais e AutoEstima. Finalmente, atendento ao segundo objectivo deste estudo, foi realizada uma ANOVA bifactorial com a autoestima em função da interacção entre os índices de segurança (apego aos pares) e os clusters pai e mãe separadamente, no intuito de perceber a existência do papel moderador do apego aos pares na auto-estima. A categorização dos índices de segurança face ao apego aos pares determinou dois níveis: seguros $(n=198)$ e inseguros $(n=195)$. Esta categorização provém de um valor obtido pela média da seguinte formulação das dimensões do IPPA (Comunicação + Confiança - Alienação), sendo mais inseguros se Mediana $\leq 7,04$ e mais seguros se Mediana> 7,04. Os resultados revelam a inexistência da interacção dos índices de segurança e dos clusters do apego na auto-estima $[F(3,325)=1,190 ; p=.314] \mathrm{e}$ $[F(3,328)=1,607 ; p=.060]$, respectivamente para o pai e mãe, o que implica que não existe um papel moderador do apego aos pares na ligação entre o apego aos pais e a auto-estima.

Preditores Relacionais da Auto-Estima. Na quarta e última parte deste estudo, foi realizada uma análise de regressão múltipla hierárquica face à auto-estima. Segundo o método hierárquico cada uma das variáveis determinam resultados onde as variáveis anteriores foram controladas pelo que permite obter resultados mais criteriosos comparativamente a uma regressão simples onde apenas uma variável é testada enquanto preditora de um resultado. Esta análise permite encarar os resultados na sua aceção de modelo global onde se encontram inerentes todas as variáveis independentes, pelo que a análise não se limita a um resultado parcelar, mas sim a uma análise integrativa onde são tidos em conta os efeitos de todas as variáveis para a auto-estima (Cohen, Cohen, West, \& Alken, 2003). Na realização desta análise foram introduzidos quatro blocos no intuito de averiguar a importância respectivamente do apego ao pai e à mãe, do apego aos pares, do conflito interparental e do gênero na auto-estima do adolescente. No bloco 1 foi introduzido o gênero explicando $1,5 \%$ da variância total $\left(R^{2}=.015\right)$, e contribuindo individualmente com $1,5 \%$ da variância para o modelo $\left(R^{2}\right.$ change $\left.=.015\right)$. No bloco 2 , o conflito interparental explicou 5,9\% da variância total $\left(R^{2}=.059\right)$, apresentando um contributo individual de $4,3 \%\left(R^{2}\right.$ change $=.043)$. No bloco 3 a entrada do apego aos pares explicou $14,4 \%$ da variância total $\left(R^{2}=.144\right)$, contribu- 
indo individualmente com $8,5 \%$ da variância para o modelo ( $R^{2}$ change=.085). Por último, no bloco 4 as dimensões de apego ao pai e à mãe (foi realizada previamente uma média entre os dois dada a necessidade de controlar a multicolinearidade), explicam $26,2 \%$ do modelo total $\left(R^{2}=.262\right)$, contribuindo individualmente com $11,8 \%$ da variância para o modelo $\left(R^{2}\right.$ change $\left.=.118\right)$. Analisando individualmente o efeito de cada uma das variáveis independentes dos blocos, constatou-se que cinco variáveis apresentam uma contribuição significativa $(p<.05)$. Entre elas, aponta-se por ordem de importância: a Qualidade do Laço Emocional Pai/Mãe ( $\beta=.284)$; Alienação no apego aos pares $(\beta=-.240)$; o Conflito Interparental ( $\beta=-.209)$; a Inibição da Exploração e Individualidade Pai/Mãe $(\beta=.205)$; e o Gênero $(\beta=.124)$ (ver Tabela 3).

Tabela 3

Regressão Múltipla Hierárquica para a Auto-estima

\begin{tabular}{lccccc}
\hline & $\mathrm{R}^{2}$ & $\mathrm{R}^{2}$ Change & $\mathrm{B}$ & $\mathrm{SE}$ & $\beta$ \\
\hline $\begin{array}{l}\text { Bloco 1 } \\
\text { Género } \\
\text { Bloco 2 }\end{array}$ & .015 & .015 & .210 & .090 & .124 \\
$\begin{array}{l}\text { CPIC(resolução, intensidade, frequência) } \\
\text { Bloco 3 }\end{array}$ & .059 & .043 & -.149 & .037 & -.209 \\
$\begin{array}{l}\text { IPPAComunicaçãoAlienaçãoConfiança } \\
\text { Bloco 4 }\end{array}$ & .144 & .085 & -.195 & .042 & -.240 \\
QVPMIei pai/mãeQle pai/mãeAs pai/mãe & .262 & .118 & -.190 .271 & .049 .068 & -.205 .284 \\
\hline
\end{tabular}

Nota. $\beta$ para um nível de significância de $p<.05$. Bloco 1- Gênero; Bloco 2- Dimensões de conflito interparental (CPIC); Bloco 3- Dimensões de apego aos pares (IPPA); Bloco 4 - Dimensões de apego aos pais (QVPM).

\section{Discussão}

Os resultados apresentados vão ao encontro dos estudos reportados na literatura, pelo que a configuração familiar parece não estar diretamente associada com indicadores de bem-estar do adolescente, especificamente a auto-estima. Hetherington e Elmore (2003) sugerem que os conflitos podem ser melhores preditores de bem-estar do que o divórcio, sendo que adolescentes de famílias intactas com elevados níveis de conflito parecem sentirse mais desconfortáveis consigo próprios do que os adolescentes que vivem em famílias divorciadas com baixos níveis de conflito. É importante ressaltar que o presente estudo não contempla diferenças dentro das famílias divorciadas em que os pais permaneceram sem ou com novos companheiros e as famílias cujos pais voltaram a casar. Nesta medida, supõe-se que em famílias monoparentais os níveis de conflito interparental tendem a variar em função do processo adaptativo, o restabelecimento pessoal, emocional, social e econômico, diminuindo ao longo do tempo. Esta análise teria sido pertinente tendo em conta que o presente estudo compreende um espaço de divórcio entre os 0 e os 18 anos de idade dos adolescentes, bem como a análise das situações de recasamento onde o conflito entre os pais pode ser diferente das famílias monoparentais, relacionando-se com o ajustamento pessoal dos pais e o espaço de tempo em que houve uma nova organização das relações (Amato \& Sobolewski, 2001). Destaca-se, contudo, que independentemente da condição atual dos adolescentes de famílias divorciadas, pretendeu-se refletir em torno da associação usualmente atribuída ao divórcio no que concerne ao bem-estar dos jovens. Na presente amostra, o conflito interparental prediz diferenças na auto-estima, mas apenas em famílias intactas, fato que poderá estar associado ao reduzido número de famílias divorciadas incluídas neste estudo. A análise com as dimensões relativas aos pares e à autoestima em função dos estilos de apego aos pais indicam que para ambos os pais, a auto-estima é significativamente elevada em adolescentes com um estilo Seguro, comparativamente aos que têm um estilo Preocupado, Amedrontado e Desinvestido. McCormick e Kennedy (1994) apontam a importância da qualidade do apego com as figuras parentais e a relação entre esses modelos de apego e a atual auto-estima dos adolescentes, obtendo resultados similares. Também Huntsinger e Luecken (2004) avaliaram como os estilos de apego parental se relacionam com o comportamento saudável dos jovens adultos, assim como o potencial papel mediador da auto-estima, concluindo que indivíduos com um estilo Seguro tinham uma elevada auto-estima e participavam de forma saudável nas interações quando comparados com indivíduos com outros estilos de apego. $\mathrm{O}$ apego aos pais apresentou ainda diferenças na qualidade do apego aos pares conforme a direcção prevista. Torna-se interessante notar que adolescentes com um estilo Preocupado (para além do Amedrontado) face ao pai reportam sentimentos de maior Alienação do que os adolescentes de estilo Seguro. Na relação com a mãe, adolescentes com um estilo Preocupado, apresentam níveis superiores de Comunicação face aos pares quando comparados com os adolescentes com um apego Desinvestido e Amedrontado. 
Estes resultados permitem perceber a dinâmica diferencial do apego aos pais e o estabelecimento das relações com os pares atendendo ao gênero das figuras parentais. Lieberman, Doyle e Markiewicz (1999) salientam a importância do apego seguro ao pai e à mãe no desenvolvimento dos adolescentes e em especial na relação com os pares. As conclusões encontradas pelos autores vêm ao encontro do presente estudo dado que em ambos os gêneros e em várias idades a qualidade da relação com ambas as figuras parentais permitiu o desenvolvimento de competências para estabelecer relações no contexto dos pares. O papel moderador do apego aos pares face à auto-estima não se observou, tal como seria esperado. Assim, embora a relação com os pares possa ser considerada relevante para a adaptação e desenvolvimento psicossocial do adolescente, a qualidade de apego aos pais parece ser um preditor mais consistente na estabilidade do adolescente, nomeadamente na auto-estima, tal como reportam os resultados da análise de regressão múltipla realizada neste estudo. Helsen, Vollebergh e Meeus (2000) suportam a ideia de que durante a adolescência o apoio parental constitui o melhor indicador da satisfação emocional. O efeito do apoio dos pares parece depender significativamente do nível de perceção de suporte parental. Nesta medida, o apoio dos pares parece não ser capaz de compensar a falta do suporte emocional dos pais, o que significa que a percepção do apoio dos pares está altamente correlacionado com a qualidade do apego às figuras parentais. Finalmente, as análises de regressão múltipla vêm confirmar alguns dos resultados apresentados ao longo deste estudo. A auto-estima mostrou-se sensível a variáveis como: a qualidade do laço emocional pai/ mãe; a alienação no apego aos pares; o conflito interparental; a inibição da exploração e individualidade pai/ mãe; e o género. Em suma, tal como esperado, os resultados indicam que as variáveis de apego associadas a uma elevada qualidade dos laços aos pais e pares têm uma crescente importância na predição da auto-estima, quando comparados com a estrutura familiar do adolescente. O conflito interparental e a forma como os pais lidam com o conflito constitui um importante preditor do ajustamento (e.g. Cummings \& Davies, 1994; Grych \& Fincham, 1990, 1993). Indubitavelmente a qualidade da relação com as figuras parentais, nomeadamente uma qualidade do laço emocional positiva e uma baixa inibição da exploração e individualidade parece assumir especial relevância, recriando nos jovens sentimentos de segurança, auto-estima e confiança em si mesmos, fato que se repercute no desenvolvimento do bem-estar dos adolescentes (Booth \& Amato, 2001).

\section{Considerações Finais}

Como abordagem final ressaltamos que o presente estudo mostrou pertinência na exploração de uma temática que se prende com a qualidade do bem-estar dos adoles- centes face ao divórcio dos pais. Nesta medida, tal como temos vindo a constatar em numerosos estudos internacionais, também com participantes portugueses existe uma crescente valorização da qualidade das relações com as figuras parentais, não havendo necessariamente alterações desadaptativas no desenvolvimento dos jovens perante a rutura marital dos pais. O conflito interparental é uma variável que mostra relevância neste processo, fato pelo qual tem vindo a ser estudado não só nas famílias divorciadas, mas também nas famílias intactas. Assim, muito embora o conflito interparental tenha sido percebido neste estudo enquanto variável que interfere na adaptação psicossocial dos adolescentes, nomeadamente na auto-estima, reconhecemos algumas limitações que se prenderam com o número reduzido de participantes provindos de famílias divorciadas, o que pode ter impedido a constatação de diferenças significativas entre adolescentes de famílias divorciadas com baixo conflito interparental e os adolescentes de famílias intactas com elevado conflito interparental. Seria pertinente em estudos futuros realizar por isso uma abordagem com uma amostra mais alargada e equitativamente distribuída sob o ponto de vista da configuração familiar; ao mesmo tempo seria interessante perceber a forma como o conflito interparental influencia o desenvolvimento de outros indicadores de adaptação psicossocial como o coping e as competências sociais.

\section{Referências}

Allen, J. P., MacElhaney, K. B., Land, D. J., Kupermic, G. P., Moore, C. W., O'Beeirne Kelly, H. (2003). A secure base in adolescence: Markers of attachment security in the motheradolescent relationship. Child Development, 74, 292-307.

Amato, P. R., \& Sobolewski, J. M. (2001). The effects of divorce and marital discord on adult children's psychological well- being. American Sociological Review, 66, 900-921.

Andrade, R., \& Matos, P. M. (2003). Childrens' Perception of Interparental Conflict Scale. Manuscrito não-publicado, Faculdade de Psicologia e de Ciências de Educação, Universidade do Porto, Portugal.

Armsden, G. C., \& Greenberg, M.T., (1987). The Inventory of Parent and Peer Attachment: Individual differences and their relationship to psychological well-being in adolescence. Journal of Youth and Adolescence, 16, 427-451.

Bartholomew, K. (1990). Adult avoidance of intimacy: An attachment perspective. Journal of Social and Personal Relationships, 7, 147-178.

Bartholomew, K., \& Horowitz, L. M. (1991). Attachment styles among young adults: A test of a four-category model. Journal of Personality and Social Psychology, 61, 226-244.

Booth, A., \& Amato, P. R. (2001). Parental predivorce relations and offspring postdivorce well-being. Journal of Marriage and Family, 63, 197-212.

Bowlby, J. (1980). Attachment and Loss: Vol. 3. New York: Basic Books.

Cohen, J., Cohen, P., West, S. G., \& Alken, L. S. (2003). Applied multiple regression/correlation analysis for the behavioural sciences (3nd ed.). Hillsdale, NJ: Lawrence Erlbaum. 
Cox, M. J., Paley, B., \& Hater, C. (2001). Interparental conflict and parent: Child relationship. In J. H. Grych \& F. D. Fincham (Eds.), Interparental conflict and child development: Theory, research and application (pp. 249-272). Cambridge, MA: Cambridge University Press.

Cummings, E. M., \& Davies, P. (1994). Children and marital conflict: The impact of family dispute and resolution. New York: Guilford.

Emery, R. E., \& Forehand, R. (1996). Parental divorce and children's well-being: A focus on resilience. In R. J. Haggerty, L. R. Sherrod, N. Garnezy, \& M. Rutter (Eds.), Stress, risk, and resilience in children and adolescents: Processes, mechanisms, and interventions. Cambridge, MA: University Press Cambridge.

Ferreira, M., \& Costa, M. E. (1998). Adaptação do Inventory of Peer and Parental Attachment - IPPA à população portuguesa. In M. Ferreira, Representação da relação de vinculação com pais e pares e gravidez na adolescência. Dissertação de Mestrado não-publicada, Faculdade de Psicologia e Ciências da Educação, Universidade do Porto, Portugal.

Goodman, G. S., \& Emery, R. E. (1998). Developmental psychology and divorce, child maltreatment, foster care and adoption. In W. Danon, I. E. Singel, \& K. Ane Reminger (Eds.), Handbook of child psychology: Vol. 4. Child psychology in practice (5th ed.). New York: John Willy \& Sons.

Grych, J. H., \& Fincham, F. D. (1990). Marital conflict and children's adjustment: A cognitive-contextual framework. Psychological Bulletin, 108, 267-290.

Grych, J. H., \& Fincham, F. D. (1993). Children's appraisals of marital conflict: Initial investigations of the cognitivecontextual framework. Child Development, 63, 215-230.

Grych, J. H., Seid, M., \& Fincham, F. D. (1992). Assessing marital conflict for the child's perspective: The children's perception of interparental conflict scale. Child Development, 63, 558-572.

Hair, J. F., Anderson, R. E., Tatham, R. L., \& Black, W. C. (1998). Multivariate data analysis. Hillsdale, NJ: PrenticeHall.

Helsen, M., Vollebergh, W., \& Meeus, W. (2000). Social support from parents and friends and emotional problems in adolescence. Journal of Youth and Adolescence, 29, 319335.

Hetherington, E. M., \& Elmore, A. M. (2003). Risk and resilience in children coping with their parents' divorce and remarriage. In S. S. Luthar (Eds.), Resilience and vulnerability: Adaptation in the context of childhood adversities. New York: Cambridge University Press.

Huntsinger, E. T., \& Luecken, L. J. (2004). Attachment relationships and health behaviour: The mediational role of self-esteem. Psychology and Health, 19, 515-526.

Lieberman, M., Doyle, A., \& Markiewicz, D. (1999). Developmental patterns in security of attachment to mother and father in late childhood and early adolescence: Associations with peer relations. Child Development, 1, 203-213.

Matos, P. M. (2002). (Des)Continuidades na vinculação aos pais e ao par amoroso no adolescente. Tese de Doutorado não-publicada, Faculdade de Psicologia e de Ciências da Educação, Universidade do Porto, Portugal.

Matos, P. M., \& Costa, M. E. (1996). Vinculação e processos desenvolvimentais em jovens e adultos. Cadernos de Consulta Psicológica, 12, 45-54.
Matos, P. M., \& Costa, M. E. (2001). The Father-Mother Attachment Questionnaire. Unpublished manuscript, Faculty of Psychology and Education, University of Porto, Portugal.

Matos, P. M., \& Costa, M. E. (2004). Significações da perda e processo de luto no divórcio. Psychologica, 35, 11-24.

McCormick, C. B., \& Kennedy, J. H. (1994). Parent-child attachment working models and self-esteem in adolescence. Journal of Youth and Adolescence, 23, 1-18.

Noller, P. (1994). Relationship with parents in adolescence: Process and outcome. In R. Montemayor, G. R. Adams, \& T. P. Gullotta (Eds.), Personal relationships during adolescence (pp. 37-77). Thousand Oaks, CA: Sage.

Peixoto, F. (2004). Qualidade das relações familiares, autoestima, auto-conceito e rendimento académico. Análise Psicológica, 1, 235-244.

Rocha, M., \& Matos, P. M. (2003). Rosenberg's Self-Esteem Scale. Adaptação para a população portuguesa. Manuscrito não-publicado, Faculdade de Psicologia e de Ciências da Educação, Universidade do Porto, Portugal.

Rosenberg, M. (1979). Conceiving the self. New York: Basic Books.

Torres, A. C. (1996). Divórcio em Portugal. Ditos e interditos. Uma análise sociológica. Oeiras, Portugal: Celta.
Recebido: $13 / 02 / 2008$

$1^{a}$ revisão: $31 / 05 / 2008$

$2^{a}$ revisão: 07/06/2008

$3^{a}$ revisão: $12 / 09 / 2008$

$4^{a}$ revisão: 04/10/2008

Aceite final: $08 / 11 / 2008$ 